\title{
Extended Wiener Process in Nonstandard Analysis
}

\author{
Shuya Kanagawa', Kiyoyuki Tchizawa ${ }^{2}$ \\ ${ }^{1}$ Department of Mathematics, Tokyo City University Setagaya, Tokyo, Japan \\ ${ }^{2}$ Institute of Administration Engineering, Ltd., Chiyoda-ku, Tokyo, Japan \\ Email: skanagaw@tcu.ac.jp, tchizawa@kthree.co.jp
}

How to cite this paper: Kanagawa, S. and Tchizawa, K. (2020) Extended Wiener Process in Nonstandard Analysis. Applied Mathematics, 11, 247-254.

https://doi.org/10.4236/am.2020.113019

Received: January 23, 2020

Accepted: March 15, 2020

Published: March 18, 2020

Copyright (c) 2020 by author(s) and Scientific Research Publishing Inc. This work is licensed under the Creative Commons Attribution International License (CC BY 4.0).

http://creativecommons.org/licenses/by/4.0/ (c) (i) Open Access

\begin{abstract}
Standing on a different view point from Anderson, we prove that the extended Wiener process defined by Anderson satisfies the definition of the Wiener process in standard analysis, for example the Wiener process at time $t$ obeys the normal distribution $N(0, t)$ by showing the central limit theorem. The essential theory used in the proof is the extended convolution property in nonstandard analysis which is shown by Kanagawa, Nishiyama and Tchizawa (2018). When processing the extension by non-standardization, we have already pointed out that it is needed to proceed the second extension for the convolution, not only to do the first extension for the delta function. In Section 2, we shall introduce again the extended convolution as preliminaries described in our previous paper. In Section 3, we shall provide the extended stochastic process using a hyper number $N$, and it satisfies the conditions being Wiener process. In Section 4, we shall give a new proof for the non-differentiability in the Wiener process.
\end{abstract}

\section{Keywords}

Wiener Process, Ito's Process, Stochastic Differential Equation, S-Continuity, Nonstandard Analysis

\section{Introduction}

Anderson [1] provided stochastic processes in nonstandard analysis to show that for $f: \Omega \rightarrow \mathbf{R}$ the following condition (a) is equivalent with (b),

(a) $f$ is Loeb measurable;

(b) there is a measurable function $F: \Omega \rightarrow{ }^{*} \mathbf{R}$ such that

$$
f(\omega) \approx F(\omega)
$$


for almost all $\omega \in \Omega$ (with respect to the Loeb measure $\mu_{L}$ ), where ${ }^{*} \mathbf{R}$ is a lifted space of $\mathbf{R}$ constructed from $\omega$-incomplete ultrafilter.

He proved that the measure on ${ }^{*} \mathbf{R}$ according to the process $W(t)$ defined by Definition 3.2 satisfies the above condition (b). Therefore, there exists an extended Wiener measure on ${ }^{*} \mathbf{R}$ which is of Loeb measure (Loeb [2], Hurd and Loeb [3]). The existence of the extended Winer measure means that $W(t)$ is an extended Wiener process in ${ }^{*} \mathbf{R}$ from Kolmogorov's extension theorem. Benot [4] [5] provided some applications of the extended Wiener measure to the stochastic analysis. Cutland [6] also introduced relevant applications of Loeb measure to the stochastic analysis.

On the other hand, in general, it is not easy to show the existence of the measurable function $F: \Omega \rightarrow{ }^{*} \mathbf{R}$ in (b) for stochastic processes. Therefore, we consider a scheme using extended convolution to show the existence of stochastic processes in ${ }^{*} R$ without using the above result.

As the main theorem in this paper, we shall provide the extended stochastic process $W(t)$ described newly in ${ }^{*} \mathbf{R}$. It satisfies the conditions being the Wiener process in nonstandard analysis. It will be proved in Section 3.

The extended Wiener process can be applied to construct some physical models, for example quantum mechanics, using the $\omega$-incomplete ultrafilter. We consider that our observation is done through the ultrafilter, since the nature is originally described by nonstandard numbers. Because of the above reasons, we need a nonstandard analysis for the convolution by the hyperfunction.

\section{Nonstandard Convolution by Hyperfunction}

Let $\mathcal{F}$ be the space of all locally integrable functions on $\mathbf{R}$. Define the space of all rapidly decreasing $C^{\infty}$ functions $\mathcal{S}$ by

$$
\sup _{x \in \mathbf{R}}\left|x^{m} f^{(k)}(x)\right|<\infty
$$

for any $m, k \in \mathbf{Z}_{+}$. Furthermore define the space of all slowly increasing functions $\mathcal{O}$ by for any $k \in \mathbf{Z}_{+}$there exist some positive constants $C_{k}$ and $M_{k} \in \mathbf{Z}_{+}$ such that

$$
\left|f^{(k)}(x)\right|<C_{k}(1+|x|)^{M_{k}}
$$

$f \in \mathcal{F}$ is Lebesgue integrable on any compact set $K$ such that

$$
-\infty<\int_{K} f(x) \mathrm{d} x<\infty
$$

For $f \in C^{\infty}$ there exists continuous $k$-th derivative $f^{(k)}(x)$ for each $k \geq 1$. For $f \in S \subset C^{\infty}$

$$
\sup _{x \in \mathbf{R}}\left|x^{m} f^{(k)}(x)\right|<\infty
$$

holds for each $k, m \in \mathbf{Z}_{+}$, where $\mathbf{Z}_{+}$means the space of all positive integers.

From the above description, we can easily obtain that

$$
S \subset \mathcal{O} \subset C^{\infty} \subset F
$$


For $f, g \in S$, the convolution of $f$ and $g$ is defined by

$$
f * g(x)=\int_{-\infty}^{\infty} f(s) g(x-s) \mathrm{d} s
$$

The definition of the convolution can be extended to the $k$-th convolution by

$$
f_{1} * f_{2} * \cdots * f_{k}(x)=\int_{-\infty}^{\infty}\left(f_{1} * \cdots * f_{k-1}\right)(s) f_{k}(x-s) \mathrm{d} s
$$

for each $k \in \mathbf{Z}_{+}$.

From the above definitions, we can easily obtain the next result.

Proposition 1.
1) $f * g(x)=g * f(x)$
2) $f *(g+h)(x)=g * f(x)+g * h(x)$
3) $\operatorname{supp}(f * g) \subseteq \operatorname{supp}(f) \cup \operatorname{supp}(g)$

Proposition 2. For $\varphi, \psi \in S$
1) $\varphi \psi \in S$
2) $\varphi * \psi \in S$
3) $(\varphi * \psi)^{(k)}(x)=\varphi^{(k)} * \psi(x)=\varphi * \psi^{(k)}(x)$

Let $\tilde{H}$ be the set of rapidly decreasing functions satisfying

1) $f(-x)=f(x)$

2) $\int_{-\infty}^{\infty} f(x) \mathrm{d} x=1$

Define a function $\bar{f}(t)$ by

$$
\bar{f}(t)(x)=\frac{1}{t} f\left(\frac{x}{t}\right), t \in(0,1]
$$

\section{Proposition 3.}

1) $f \in \tilde{H} \Rightarrow \bar{f}(t) \in \tilde{H}$

2) $f, g \in \tilde{H} \Rightarrow f * g \in \tilde{H}$

Example 2.1. Figure 1 gives a typical example of hyperfunction at around the origin.

\section{Extended Wiener Process in Nonstandard Analysis}

Why does the extended stochastic process in Definition 3.2 satisfy the conditions
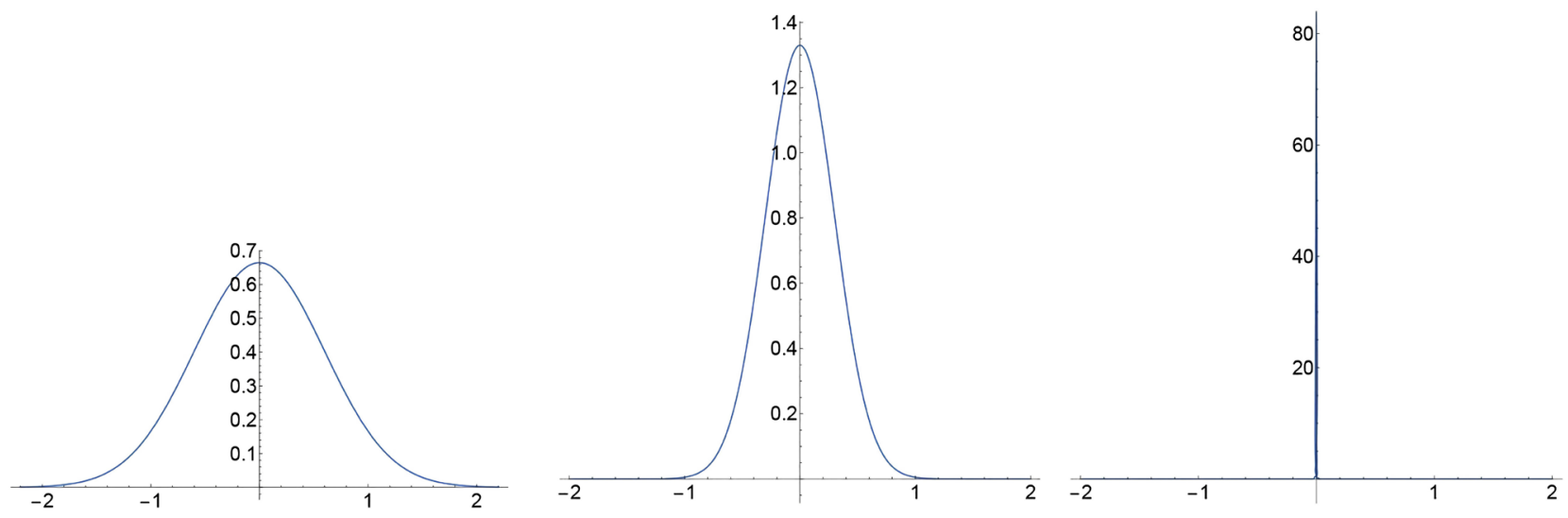

Figure 1. $N\left(0, \sigma^{2}\right), \sigma=6 / 10, \sigma=3 / 10$, hyperfunction $\sigma=1 / N, N \in * N \backslash N$. 
being Wiener process? First, let us show the definition of the extended Wiener process according to Anderson [1] [7].

Definition 3.1. For fixed $N \in *_{N} \backslash N$, put $\Delta t=N^{-1}$. The hyperfinite time line $\mathbf{T}$ based on $\Delta t$ for the interval $[0, T]$ is the set defined by

$$
\mathbf{T}=\{0, \Delta t, 2 \Delta t, \cdots, T-\Delta t, T\},
$$

for some $T>0$.

Definition 3.2. Assume that a sequence of i.i.d. random variables $\left\{\Delta W_{k}, k=1, \cdots, N T\right\}$ has the distribution

$$
P\left\{\Delta W_{k}=\sqrt{\Delta t}\right\}=P\left\{\Delta W_{k}=-\sqrt{\Delta t}\right\}=\frac{1}{2},
$$

for each $k=1, \cdots, N T$. An extended stochastic process $\{W(t), t \in \mathbf{T}\}$ is defined by

$$
W(t)=\sum_{k=1}^{N t} \Delta W_{k}, \quad t \in \mathbf{T} .
$$

We shall prove that the extended process $W(t)$ satisfies the following conditions being Wiener process.

Definition 3.3. If the process $W(t)$ satisfies the following conditions, then $W(t)$ is called the Wiener process.

1) $W(0)=0$. a.s.

2) $W(t)$ is a continuous function with probability 1 .

3) $W(t)$ has stationary, independent increments.

4) The increment $W(t+s)-W(s)$ has the normal distribution $N(0, t)$ for any $s, t \geq 0$.

Theorem 3.4. The extended process $W(t)$ satisfies the conditions in Definition 3.3.

Proof)

Now, we go back the distribution of $W(t)$. From the nonstandardization of the convolution in the previous section, we calculate the characteristic function (Fourier transform) of $W(t)$.

$$
P\{W(t) \leq x\}=P\left\{\sum_{k=1}^{N t} \Delta W_{k} \leq x\right\}=\int_{-\infty}^{x} f * \cdots * f(u) \mathrm{d} u,
$$

where $f(u)$ is the density function of $\Delta W_{k}$.

$$
\begin{aligned}
E\left[\mathrm{e}^{i \theta W(t)}\right] & =E\left[\mathrm{e}^{i \theta \sum_{k=1}^{N t} \Delta W_{k}}\right]=\int_{-\infty}^{\infty} \mathrm{e}^{i \theta u} f * \cdots * f(u) \mathrm{d} u \\
& =\prod_{k=1}^{N t} \int_{-\infty}^{\infty} \mathrm{e}^{i \theta u} f(u) \mathrm{d} u=\prod_{k=1}^{N t} E\left[\mathrm{e}^{i \theta \Delta W_{k}}\right] \\
& =\prod_{k=1}^{N t} \frac{1}{2}\left\{\mathrm{e}^{-i \theta \sqrt{\Delta t}}+\mathrm{e}^{i \theta \sqrt{\Delta t}}\right\}=\prod_{k=1}^{N t} \cos (\theta \sqrt{\Delta t}) \\
& =\left(\cos \frac{\theta}{\sqrt{N}}\right)^{N t}=\mathrm{e}^{\frac{t \theta^{2}}{2}} .
\end{aligned}
$$

Note that, in standard analysis, the last term in (2) is shown by the following 
equation,

$$
\begin{aligned}
\left(\cos \frac{\theta}{\sqrt{N}}\right)^{N t} & =\lim _{n \rightarrow \infty}\left(\cos \frac{\theta}{\sqrt{n}}\right)^{n t}=\lim _{n \rightarrow \infty}\left\{1-\frac{\theta^{2}}{2 ! n}+\frac{\theta^{4}}{4 ! n^{2}}-\cdots\right\}^{n t} \\
& =\lim _{n \rightarrow \infty}\left\{1-\frac{\theta^{2}}{2 ! n}+\frac{\theta^{4}}{4 ! n^{2}}-\cdots\right\}^{n t} \\
& =\lim _{n \rightarrow \infty}\left\{1-\frac{\theta^{2}}{2 ! n}+\frac{\theta^{4}}{4 ! n^{2}}-\cdots\right\}^{n t} \\
& =\lim _{n \rightarrow \infty}\left\{\left(1-\frac{\theta^{2}}{2 n}\right)^{\frac{2 n}{\theta^{2}}}\right\}^{\frac{t \theta^{2}}{2}}=\mathrm{e}^{\frac{t \theta^{2}}{2}} .
\end{aligned}
$$

Therefore, $W(t)$ obeys the normal distribution $N(0, t)$. Furthermore, it is easy to prove that $W(t)$ has the stationary and independent increments from the Definition 3.2. Then, we can show that $W(t)$ satisfies all conditions of the Wiener process.

\section{Proof of the Non-Differentiability of Wiener Process}

In this section, we give the proof that the Wiener process has the property of non-differentiability. It is well known that Wiener process in standard analysis is non-differentiable a.s. though. The proof of the non-differentiability was shown by Dvoretski, Erdös and Kakutani [8]. (See e.g. Theorem 12.25 in Breiman [9].) On the other hand, the law of the iterated logarithm holds for the extended Wiener process as following (3) and (4). As to the law of the iterated logarithm in standard analysis, see e.g. Karatzas and Shreve [10], the Section 2.9. The original proof for i.i.d. random variables is due to Khintchine [11].

Theorem 4.1. Every sample path of Wiener process defined by

$$
W(t)=\sum_{k=1}^{N t} \Delta W_{k}, \quad t \in \mathbf{T},
$$

has the non-differentiability.

Proof)

$$
\sup _{N \in * \mathrm{NIN}} \frac{W(1 / \sqrt{N})}{\sqrt{2(1 / \sqrt{N}) \log \log (\sqrt{N})}}=1 \text {, a.s. }
$$

and

$$
\inf _{N \in \in^{* N N N}} \frac{W(1 / \sqrt{N})}{\sqrt{2(1 / \sqrt{N}) \log \log (\sqrt{N})}}=-1, \quad \text { a.s. }
$$

Since $W(t+s)-W(t)$ is also a Wiener process for some $t \in \mathbf{T}$ and any $s \in \mathbf{T}$,

$$
\sup _{N \in \epsilon^{*} \mathrm{NN}} \frac{W(t+1 / \sqrt{N})-W(t)}{\sqrt{2(1 / \sqrt{N}) \log \log (\sqrt{N})}}=1 \text {, a.s. }
$$


and

$$
\inf _{N \in *^{* N N}} \frac{W(t+1 / \sqrt{N})-W(t)}{\sqrt{2(1 / \sqrt{N}) \log \log (\sqrt{N})}}=-1, \quad \text { a.s. }
$$

for any $t>0$. Therefore

$$
\sup _{N \in *^{* N N N}} \frac{W(t+1 / \sqrt{N})-W(t)}{\sqrt{1 / \sqrt{N}}}>\sqrt{2 \log \log (\sqrt{N})}, \text { a.s. }
$$

and

$$
\inf _{N \in * \mathrm{NNN}} \frac{W(t+1 / \sqrt{N})-W(t)}{\sqrt{1 / \sqrt{N}}}<-\sqrt{2 \log \log (\sqrt{N})}, \quad \text { a.s. }
$$

(7) and (8) imply the non-differentiability of the sample path of Wiener process.

Remark 1. From Definition 3.1 for the extended Wiener process, we have

$$
\frac{W(1 / N)}{\sqrt{2(1 / N) \log \log (N)}}=\frac{\Delta W_{1}}{\sqrt{2(1 / N) \log \log (N)}}=\left\{\begin{array}{l}
\frac{1}{\sqrt{2 \log \log (N)}} \\
\frac{-1}{\sqrt{2 \log \log (N)}} .
\end{array}\right.
$$

Thus the law of the iterated logarithm does not hold for

$$
\frac{W(1 / N)}{\sqrt{2(1 / N) \log \log (N)}} \text {. }
$$

Notice that

$$
\frac{W(1 / \sqrt{N})}{\sqrt{2(1 / \sqrt{N}) \log \log (\sqrt{N})}}
$$

is defined on the hyperfinite line

$$
\sqrt{\mathbf{T}}=[0, \Delta t, 2 \Delta t, 3 \Delta t, \cdots,(N / \sqrt{N}) \Delta t] .
$$

Therefore,

$$
\sup _{N \in * \mathrm{NNN}} \frac{W(1 / \sqrt{N})}{\sqrt{2(1 / \sqrt{N}) \log \log (\sqrt{N})}}
$$

and

$$
\inf _{N \in \epsilon^{*} \mathrm{NN}} \frac{W(1 / \sqrt{N})}{\sqrt{2(1 / \sqrt{N}) \log \log (\sqrt{N})}}
$$

exist and the law of the iterated logarithm (3) and (4) hold on the hyperfinite line $\sqrt{\mathbf{T}}$.

Remark 2. From Definition 3.2 the Wiener process $W(t)$ is defined by the sum of i.i.d. random variables $\left\{\Delta W_{k}, k=1, \cdots, N T\right\}$. Therefore, we can prove the 
non-differentiability of Wiener process by the law of the iterated logarithm (3) and (4). Since the above proof in the sense of nonstandard analysis cannot be translated to the proof in standard analysis, another proof using standard one in [8] is needed to show the non-differentiability. This is a typical example of the advantage of the extended Wiener process.

\section{Conclusion}

Anderson [1] showed that the process $W(t)$ defined by Definition 3.2 satisfies the conditions (1)-(4) in Definition 3.3 of Wiener process from the equivalence of (a) and (b) due to Loeb [2]. On the other hand, we showed the extended Wiener process for $W(t)$ satisfies the conditions (1)-(4) directly by the nonstandardization of the convolution. When we extend the time space and obtain extended Wiener process, the nonstandard number $N$ is efficient enough to describe the precise structure. It is also reminded that the $N$ is applied to "extended Wiener measure" described in [12] [13]. The hyperfinite time line $\mathbf{T}$ is a key word. Notice that the delta function is described by the normal distribution in this state. Furthermore, we provided a new proof of the non-differentiability on the Wiener process using the extended law of the iterated logarithm for the Wiener process in nonstandard analysis.

\section{Supported}

The first author is supported in part by Grant-in-Aid Scientific Research (C), No. 18K03431, Ministry of Education, Science and Culture, Japan.

\section{Conflicts of Interest}

The authors declare no conflicts of interest regarding the publication of this paper.

\section{References}

[1] Anderson, R.M. (1976) A Non-Standard Representation for Brownian Motion and Ito Integration. Israel Journal of Mathematics, 25, 15-46. https://doi.org/10.1007/BF02756559

[2] Loeb, A. (1971) A Nonstandard Representation of Measurable Spaces and $L^{\infty}$. Bulletin of the American Mathematical Society, 77, 540-544. https://doi.org/10.1090/S0002-9904-1971-12745-3

[3] Hurd, A.E. and Loeb, P.A. (1985) Introduction to Nonstandard Real Analysis. Acad. Press, Cambridge.

[4] Benot, E. (1989) Diffusions discrètes et mécanique stochastique. Prépubli. Lab. Math. J. Dieudonné, Université de Nice.

[5] Benot, E. (1995) Random Walks and Stochastic Differential Equations. In: Nonstandard Analysis in Practice, Springer, Berlin, 71-90. https://doi.org/10.1007/978-3-642-57758-1 4

[6] Cutland, N.J. (2000) Loeb Measures in Practice: Recent Advances. Lecture Notes in Mathematics 1751, Springer, Berlin. https://doi.org/10.1007/b76881 
[7] Anderson, R.M. (1982) Star-Finite Representation of Measure Spaces. Transactions of the American Mathematical Society, 271, 667-687. https://doi.org/10.1090/S0002-9947-1982-0654856-1

[8] Dvoretzky, A., Erdös, P. and Kakutani, S. (1961) Nonincrease Everywhere of the Brownian Motion Process. 4th Berkeley Symposium on Mathematical Statistics and Probability, Vol. 2, 103-116.

[9] Breiman, L. (1968) Probability. Addison-Wesley Publ., Boston.

[10] Karatzas, I. and Shreve, S.E. (1991) Brownian Motion and Stochastic Calculus. 2nd Edition, Springer, Berlin.

[11] Khintchine, A. (1924) Über einen Satz der Wahrscheinlichkeitsrechnung. Fundamenta Mathematicae, 6, 9-20. https://doi.org/10.4064/fm-6-1-9-20

[12] Kanagawa, S., Nishiyama, R. and Tchizawa, K. (2018) Extended Wiener Measure by Nonstandard Analysis for Financial Time Series. Applied Mathematics, 9, 975-984. https://doi.org/10.4236/am.2018.98066

[13] Kanagawa, S. and Tchizawa, K. (2019) Proof of Ito's Formula for Ito's Process in Nonstandard Analysis. Applied Mathematics, 10, 561-567. https://doi.org/10.4236/am.2019.107039

\section{Symbols}

$\Omega$ : probability space

$\mathbf{R}$ : real numbers

${ }^{*} \mathbf{R}$ : hyper real numbers

$\mathcal{F}$ : space of all locally integrable functions on $\mathbf{R}$

$\mathcal{S}$ : space of all rapidly decreasing functions

$\mathbf{Z}_{+}$: positive natural numbers

$\mathcal{O}$ : space of all slowly increasing functions

$N$ : hyper number

$N^{-1}$ : infinitesimal

$\mathbf{N}$ : natural numbers

${ }^{*} \mathbf{N}$ : hyper natural numbers

$\mathbf{T}$ : hyperfinite time line 